\title{
Circulating VEGF as a biomarker for diagnosis of ovarian cancer: a systematic review and a meta-analysis
}

This article was published in the following Dove Press journal:

OncoTargets and Therapy

15 May 2015

Number of times this article has been viewed

\author{
Bin Liang \\ Qun $\mathrm{He}$ \\ Liansheng Zhong \\ Shaocheng Wang \\ Zhongcheng Pan \\ Tianjiao Wang \\ Yujie Zhao \\ Biochip Center and State Key Lab \\ of Cell Biology, China Medical \\ University, Shenyang, Liaoning, \\ People's Republic of China
}

Correspondence: Yujie Zhao

Biochip Center and State Key Lab

of Cell Biology, China Medical University,

Beier Road 92, Heping District, Shenyang,

Liaoning I I000I, People's Republic

of China

Email zhaoyjchip@yeah.net
Abstract: VEGF is a frequently studied angiogenic factor in ovarian cancer (OC), and is considered to have an important role in the progression of OC. However, its diagnostic value has not been widely accepted because the conclusions are inconsistent and even conflicting. Therefore, we performed a meta-analysis to evaluate the diagnostic value of VEGF in OC. A systematic literature search was conducted using the PubMed, Cochrane Library, EMBASE, Chinese National Knowledge Infrastructure, and WANFANG databases for relevant published articles (the last search update was November 18, 2014). The diagnosis sensitivity, specificity, positive likelihood ratio, negative likelihood ratio, diagnostic odds ratio, and the summary receiver operating characteristic curves were pooled by Meta DiSc 1.4 software. A total of ten studies with 1,131 subjects were finally included in this meta-analysis. The pooled sensitivity, specificity, positive likelihood ratio, negative likelihood ratio, diagnostic odds ratio, and summary receiver operating characteristic curves were 0.67 (0.63-0.73), $0.78(0.75-0.81), 3.08$ (6.36-12.22), 0.39 (0.29-0.51), 9.10 (5.43-45.25), and 0.8175, respectively. Furthermore, to explore the sources of heterogeneity, we conducted subgroup analyses based on ethnicity and sample size. The diagnostic accuracy of VEGF was higher in an Asian population than in a Caucasian population. A similar finding was found in subgroups with the smaller sample size $(<100$ subjects). In conclusion, the present meta-analysis suggests that VEGF has moderate diagnostic accuracy for OC. Considering our limitations and the heterogeneity among our selected studies, larger, well-designed prospective and multicenter validation studies are needed to evaluate the diagnostic value of serum VEGF for OC.

Keywords: gynecological tumor, diagnosis, blood, review

\section{Introduction}

Ovarian cancer (OC) is the fifth most common type of cancer in females and the leading cause of mortality for gynecological malignancies. ${ }^{1,2}$ Most OC patients are diagnosed with late-stage disease because the asymptomatic progression is poorly understood and an efficient screening strategy is not presently available. ${ }^{3}$ As a result, many OC patients are either misdiagnosed or diagnosed at later stages with poor prognosis. ${ }^{4}$ Thus, it is critical to understand the molecular mechanisms involved in OC progress and to identify early and accurate screening markers of OC is especially important for OC management.

VEGF, one of the key mediators of angiogenesis, promotes the recruitment and proliferation of endothelial cells and their precursors within the tumor, and thus plays a critical role in angiogenesis during tumor development. ${ }^{5,6}$ In 1994, Kondo et al first recognized the potential of VEGF as a serum diagnostic marker for malignant disease. ${ }^{7}$ Accumulating evidence also has demonstrated that the circulating levels of 
VEGF are elevated in various cancers, including colorectal cancer, ${ }^{8}$ lung cancer, ${ }^{9}$ gastric cancer, ${ }^{10}$ endometrial cancer, ${ }^{11}$ breast cancer, ${ }^{12}$ OC. ${ }^{13}$ Thus, VEGF is a focus of interest with respect to vascular research and oncology.

VEGF is a frequently studied angiogenic factor in $\mathrm{OC}$, and is considered to have an important role in the progression of OC. However, its diagnostic value has not been widely accepted because the conclusions are inconsistent and even conflicting. Therefore, we performed a systematic review and meta-analysis to evaluate the diagnostic value of circulating VEGF in OC, with the purpose to guide the clinical management.

\section{Materials and methods} Literature search strategy

A systematic literature search was conducted using the PubMed, Cochrane Library, EMBASE, Chinese National Knowledge Infrastructure, and WANFANG databases for relevant published articles (the last search update was November 18, 2014). Search terms "ovarian cancer", "ovarian tumor”, “gynecological tumor”; “blood”, “serum”, “plasma”, "circulating"; "diagnosis"; and "sensitivity and specificity" were used individually and in various pairwise combinations. All eligible studies were retrieved, and their bibliographies were checked for other relevant publications.

\section{Study selection criteria}

Studies were included according to following criteria: 1) studies regarding the diagnostic potential of circulating VEGF for OC; 2) studies with a gold reference standard for OC diagnosis; 3 ) sensitivity (SEN) and specificity (SPE) of VEGF were reported to provide sufficient information to construct $2 \times 2$ contingency tables. Exclusion criteria were as follows: 1 ) incomplete data to construct $2 \times 2$ contingency tables; 2) duplicate studies; 3 ) reviews, letters and comments; and 4) low-quality articles.

\section{Quality assessment}

Two reviewers (Liang and $\mathrm{He}$ ) independently assessed the quality of included studies by using the QUADAS (Quality Assessment of Diagnosis Accuracy Studies) criteria, which was developed as a validated instrument for diagnostic studies. ${ }^{14}$ QUADAS criteria includes 14 questions, each of which is scored as yes, no, or unclear ("yes" means " 1 " score, "no, or unclear" means "0" score), and the total scores were 14.

\section{Data extraction}

All studies meeting the inclusion criteria were independently assessed by a pair of reviewers (Liang and Zhong).
Disagreements were discussed and resolved by consensus. The following data were extracted from each study: first author's name, publication year, country, ethnicity, sample size (number of cases and controls), method of laboratory measurements of VEGF, cut-off values, and diagnostic performance (SEN, SPE, true positive, false positive, false negative, and true negative), and QUADAS scores. Moreover, we conducted subgroup analysis by ethnicity (Asian and Caucasian) and sample size ( $\geq 100$ subjects and $<100$ subjects).

\section{Statistical analyses}

The STATA 12.0 software (StataCorp LP, College Station, TX, USA) and Meta-Disc 1.4 (XI Cochrane Colloquium, Barcelona, Spain) were used to perform all data analysis. In the meta-analysis, we used a bivariate regression approach to calculate the pooled SEN and SPE, positive and negative likelihood ratios (PLR and NLR, respectively), and summary receiver operating characteristic (SROC) curves to summarize the study results, as well as their corresponding $95 \%$ confidence intervals $\left(95 \%\right.$ CIs). The $Q$ and $I^{2}$ statistics were used to assess heterogeneity, where $P \leq 0.05$ or $I^{2}>50 \%$ were considered to indicate significant heterogeneity. When the result of the $Q$-test and $I^{2}$ statistics suggested heterogeneity ( $P \leq 0.05$ and $I^{2}>50 \%$ ), a random-effects model (DerSimonian-Laird method) was used; otherwise, fixed-effects model (Mantel-Haenszel method) was adopted. Moreover, analysis of diagnostic threshold effects was quantified by the Spearman correlation coefficient. Publication bias was assessed by visual inspection of Deek's funnel plots which might affect the validity of the estimates. The symmetry of the Deek's funnel plot was further evaluated by Egger's linear regression test. ${ }^{15} P$-values for all comparisons were two-tailed, and statistical significance was defined as a $P$-value less than 0.05 .

\section{Results}

\section{Baseline characteristics of included studies}

The process of study selection was shown in Figure 1. We initially identified 1,071 candidate articles reporting results regarding circulating VEGF levels and the risk of OC. Of the 1,071 studies, 1,035 were excluded following a preliminary review, leaving 36 studies for detailed full-text evaluation. Finally, a total of ten studies with 478 cases and 653 controls were included for systematic review and meta-analysis. The characteristics of the included studies were shown in Table $1 .{ }^{16-25}$ Of ten studies, six studies were conducted in 


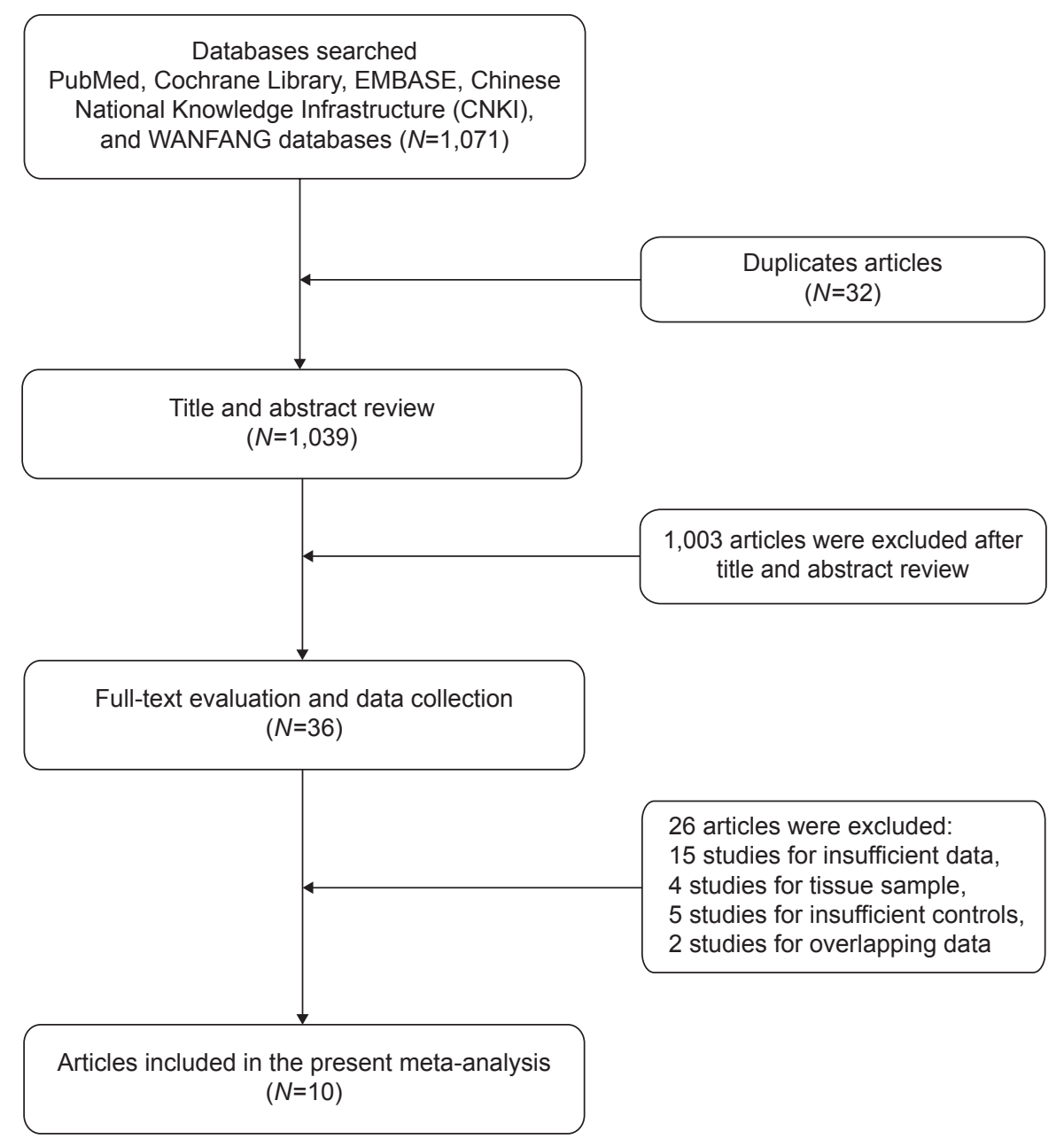

Figure I Flowchart of studies included in meta-analysis.

Western countries, and four studies were conducted in Asian countries. Circulating VEGF was measured with enzymelinked immunosorbent assay (ELISA) in all included studies. All studies were published from 1998 to 2013, and the sample sizes ranged from 60 to 260 subjects.

\section{Quality assessment of the included studies}

The ten studies were scored by QUADAS method by two independent reviewers. All included studies had QUADAS scores $\geq 10$, suggesting that the quality of included studies was generally high and satisfied the majority of the criteria. The study characteristics, along with QUADAS scores, were presented in Table 1.

\section{Heterogeneity assessment and threshold effect}

As shown by the forest plot of SEN, SPE, PLR, NLR, and diagnostic odds ratio (DOR) for VEGF levels in OC, the heterogeneity analysis revealed $I^{2}$ values of $82.1 \%(P<0.001)$ for SEN, 84.7\% $(P<0.001)$ for SPE, 74.2\% $(P<0.001)$ for PLR, 70.8\% $(P<0.001)$ for NLR, and 63.2\% $(P=0.004)$ for DOR. This provided evidence of high levels of heterogeneity in the ten studies. However, the Spearman correction coefficient was $0.321(P=0.365)$, indicating heterogeneity was not caused by the threshold effect. Thus, the random effects model approach was used in this meta-analysis to eliminate heterogeneity.

\section{Quantitative data synthesis}

The pooled SEN of VEGF for OC diagnosis was 0.67 (95\% CI: 0.63-0.71) (Figure 2A) and the pooled SPE was 0.78 (95\% CI: 0.75-0.81) (Figure 2B). The PLR was 3.08 (95\% CI: 2.27-4.19) (Figure 3A), the NLR was 0.39 (95\% CI: 0.29-0.51) (Figure 3B), and the DOR was 9.10 (95\% CI: 5.43-15.25) (Figure 3C). The SROC curve shows an overall summary of tests, which illustrates the relationship between SEN and SPE. The bivariate SROC curves showed area 


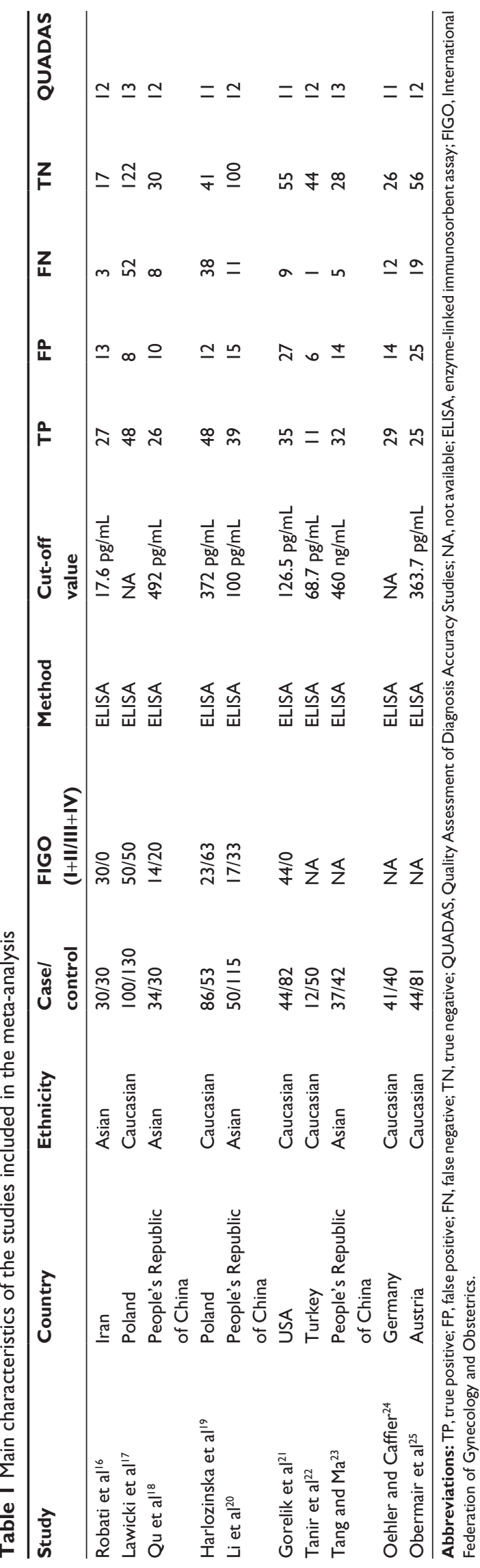

under the curve (AUC) of circulating VEGF was 0.8175, suggesting that efficiency of VEGF for OC diagnosis was considerable (Figure 4).

Subgroup analyses were also conducted according to the ethnicity and sample size to explore the source of heterogeneity. The assessment results of VEGF diagnostic accuracy in each subgroup were shown in Table 2. The subgroup analyses on ethnicity show that the SEN was 0.82 (95\% CI: 0.75-0.88), the SPE was 0.77 (95\% CI: 0.71-0.82), the PLR was 3.12 (95\% CI: 1.97-4.94), the NLR was 0.25 (95\% CI: 0.18-0.36), the DOR was 14.95 (95\% CI: 8.76-25.50), and the AUC was 0.8705 for Asian population. By comparison, the corresponding SEN, SPE, PLR, NLR, DOR, and AUC of the Caucasian population were 0.60 (95\% CI: $0.54-0.65$ ), 0.79 (95\% CI: 0.75-0.83), 3.11 (95\% CI: 1.99-4.85), 0.51 (95\% CI: 0.41-0.64), 7.00 (95\% CI: 3.58-13.70), and 0.7814, respectively. Therefore, the diagnostic accuracy of VEGF was higher in an Asian population than in a Caucasian population.

A similar finding was found in the subgroup with the smaller sample size ( $<100$ subjects). For the studies with smaller sample size, the SEN was 0.81, the SPE was 0.72, and the AUC was 0.8113 , respectively. While the studies with a larger sample size revealed that the SPE increased, but the SEN decreased significantly.

\section{Publication bias}

Deek's funnel plot and Egger's test were performed to assess the publication bias in the literature. In spite of a little asymmetry observed in Deek's funnel plot, the Egger's test demonstrated that there is no evidence of publication bias ( $P=0.799)$.

\section{Discussion}

OC remains a feared disease as non-specific symptoms result in delayed diagnosis and presentation with late-stage disease. To date, there is still a great need to improve early detection methods and to identify new diagnostic biomarkers.

VEGF, one of the most directly functional and powerful growth factors currently found, is an indispensable part for tumor growth, invasion, and metastasis. ${ }^{26}$ Based on the evidence that VEGF plays central roles in angiogenic processes in cancer, various VEGF signal inhibitors, including anti-VEGF neutralizing antibodies and VEGFR kinase/multikinase inhibitors, have been successfully developed and are now widely used in cancer therapy. So far, there are many studies dedicated to the relationship between circulating 


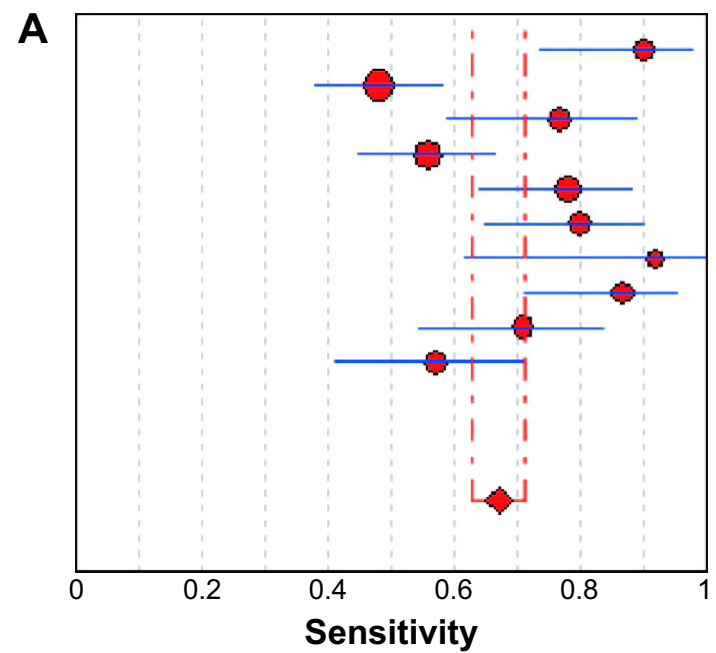

\section{B}

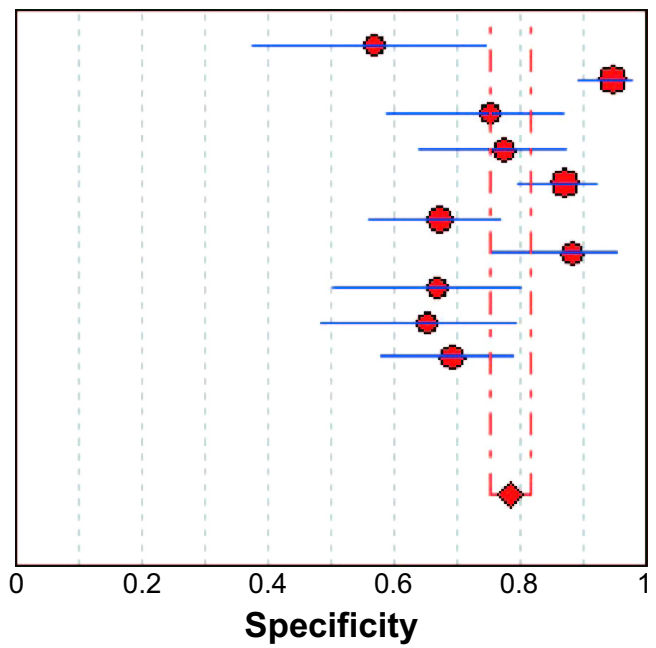

Sensitivity $(95 \% \mathrm{Cl})$

Robati et $\mathrm{al}^{16} \quad 0.90(0.73-0.98)$

Lawicki et al ${ }^{17} \quad 0.48(0.38-0.58)$

Qu et $\mathrm{al}^{18} \quad 0.76(0.59-0.89)$

Harlozinska et al ${ }^{19} \quad 0.56(0.45-0.67)$

$\mathrm{Li}$ et $\mathrm{al}^{20} \quad 0.78(0.64-0.88)$

Gorelik et al $^{21} \quad 0.80(0.65-0.90)$

Tanir et al22 $\quad 0.92(0.62-1.00)$

Tang and $\mathrm{Ma}^{23} \quad 0.86(0.71-0.95)$

Oehler and Caffier ${ }^{24} \quad 0.71(0.54-0.84)$

Obermair et al ${ }^{25} \quad 0.57(0.41-0.72)$

Pooled sensitivity $=0.67(0.63-0.71)$

$\chi^{2}=50.30 ; d f=9(P=0.0000)$

Inconsistency $\left(I^{2}\right)=82.1 \%$

Robati et al ${ }^{16}$

Lawicki et al ${ }^{17}$

Qu et al ${ }^{18}$

Harlozinska et al ${ }^{19}$

Li et $\mathrm{al}^{20}$

Gorelik et $\mathrm{al}^{21}$

Tanir et al2 ${ }^{22}$

Tang and $\mathrm{Ma}^{23}$

Oehler and Caffier ${ }^{24}$

Obermair et $\mathrm{al}^{25}$

Specificity $(95 \% \mathrm{Cl})$

$0.57(0.37-0.75)$

$0.95(0.89-0.98)$

$0.75(0.59-0.87)$

$0.77(0.64-0.88)$

$0.87(0.79-0.93)$

$0.67(0.56-0.77)$

$0.88(0.76-0.95)$

$0.67(0.50-0.80)$

$0.65(0.48-0.79)$

$0.69(0.58-0.79)$

Pooled specificity $=0.78(0.75-0.81)$

$\chi^{2}=58.68 ; d f=9(P=0.0000)$

Inconsistency $\left(I^{2}\right)=84.7 \%$

Figure 2 Forest plots (random effects model) of pooled sensitivity and specificity of each included study for diagnosis of ovarian cancer.

Notes: (A) Sensitivity; (B) specificity.

Abbreviation: $\mathrm{Cl}$, confidence interval.

VEGF levels and tumor behaviors of OC patients, but the results are inconsistent. To uncover the epidemiological features of VEGF and its diagnostic significance in OC, we performed a comprehensive analysis of the performance of circulating VEGF as a biomarker to diagnose OC.

The present meta-analysis showed that the pooled characteristics were as follows: SEN of 0.67, SPE of 0.782, PLR of 3.08 , and NLR of 0.39 . The results indicated that the diagnostic accuracy may not be high enough as expected. However, VEGF still has moderate diagnostic value compared with traditional biomarkers, such as CA125 and HE4, (with sensitivities of $74.0 \%$ and $74.0 \%$ for diagnosing OC respectively). ${ }^{27}$ DOR, ranging from 0 to $\infty$, is commonly used to evaluate test performance from a statistical point of view because it represents a single indicator of diagnostic test accuracy that combines SEN and SPE data. ${ }^{28}$ We calculated that the DOR of VEGF was 9.10 (95\% CI: 5.43-15.25), indicating that the odds for positive VEGF results among subjects with OC was nine times higher than the odds for positive VEGF results among subjects without OC. This result further confirms that VEGF is a useful marker for the diagnosis of OC. The ideal SROC curves present a global summary of test performance and reveal the equivalency between SEN and SPE, and its position is near the upper-left corner, which would indicate a best performance. ${ }^{29}$ Our results indicated that the AUC was 0.8175 , suggesting that the level of overall accuracy of the circulating VEGF is acceptable.

Heterogeneity is a potential problem when consequences are interpreted in a meta-analysis. In the present meta-analysis, significant heterogeneity was detected among 
A

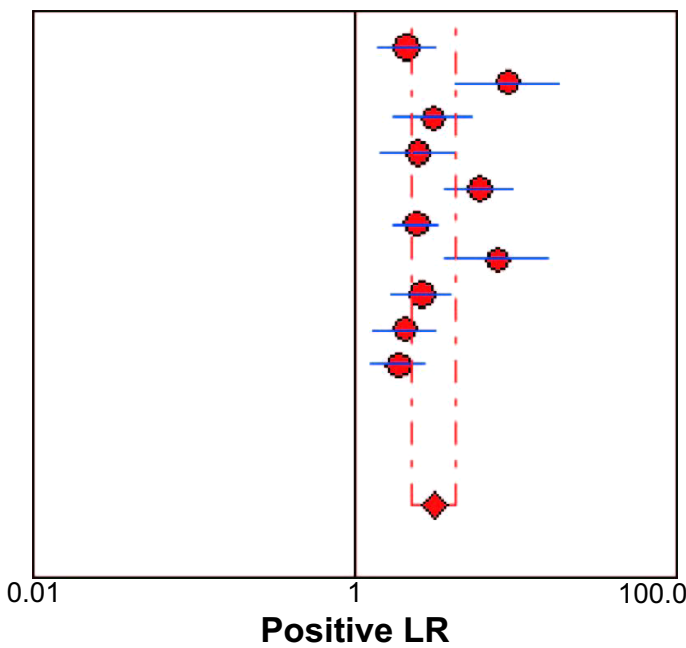

Robati et $\mathrm{al}^{16}$

Lawicki et al ${ }^{17}$

Qu et al ${ }^{18}$

Harlozinska et al ${ }^{19}$

Li et $\mathrm{al}^{20}$

Gorelik et al ${ }^{21}$

Tanir et $\mathrm{al}^{22}$

Tang and $\mathrm{Ma}^{23}$

Oehler and Caffier ${ }^{24}$

Obermair et $\mathrm{al}^{25}$
Positive LR (95\% Cl)

$2.08(1.36-3.18)$

$8.85(4.18-18.70)$

$3.06(1.73-5.40)$

$2.47(1.45-4.20)$

$5.98(3.65-9.80)$

$2.42(1.71-3.41)$

$7.64(3.54-16.49)$

$2.59(1.66-4.05)$

$2.02(1.27-3.22)$

$1.84(1.22-2.79)$

Random effects model

Pooled positive LR =3.08 (2.27-4.19)

Cochran- $Q=34.84 ; d f=9(P=0.0001)$

Inconsistency $\left(I^{2}\right)=74.2 \%$

$\tau^{2}=0.1752$

B

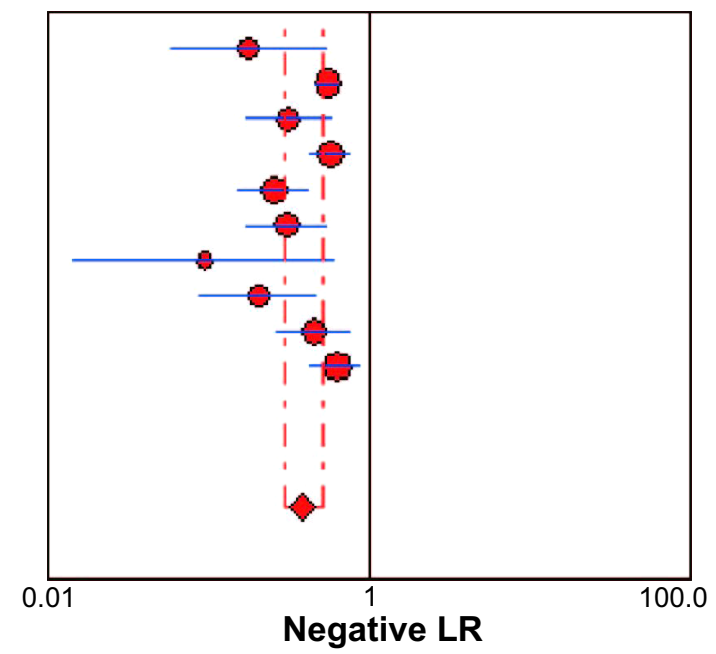

Negative LR $(95 \% \mathrm{Cl})$

Robati et al ${ }^{16}$

Lawicki et al ${ }^{17}$

Qu et al ${ }^{18}$

Harlozinska et al ${ }^{19}$

$\mathrm{Li}$ et $\mathrm{al}^{20}$

Gorelik et $\mathrm{al}^{21}$

Tanir et $\mathrm{al}^{22}$

Tang and $\mathrm{Ma}^{23}$

Oehler and Caffier ${ }^{24}$

Obermair et $\mathrm{al}^{25}$

$0.18(0.06-0.54)$

$0.55(0.45-0.67)$

$0.31(0.17-0.59)$

$0.57(0.43-0.75)$

$0.25(0.15-0.43)$

$0.30(0.17-0.56)$

$0.09(0.01-0.62)$

$0.20(0.09-0.47)$

$0.45(0.27-0.76)$

$0.62(0.43-0.90)$

Random effects model

Pooled negative LR $=0.39(0.29-0.51)$

Cochran- $\mathrm{Q}=30.78 ; d f=9 \quad(P=0.0003)$

Inconsistency $\left(I^{2}\right)=70.8 \%$

$\tau^{2}=0.1227$

C

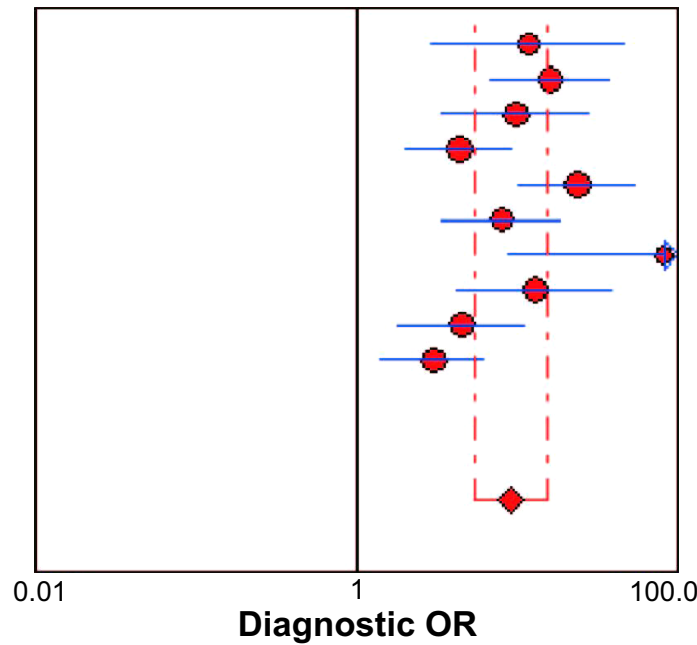

Robati et al ${ }^{16}$

Lawicki et al ${ }^{17}$

Qu et al ${ }^{18}$

Harlozinska et al ${ }^{19}$

Li et $\mathrm{al}^{20}$

Gorelik et $\mathrm{al}^{21}$

Tanir et $\mathrm{al}^{22}$

Tang and $\mathrm{Ma}^{23}$

Oehler and Caffier ${ }^{24}$

Obermair et $\mathrm{al}^{25}$
Diagnostic OR $(95 \% \mathrm{Cl})$

$11.77(2.92-47.46)$

$16.09(6.83-37.90)$

$9.75(3.35-28.36)$

$4.32(2.00-9.33)$

$23.64(9.99-55.94)$

7.92 (3.33-18.82)

$80.67(8.78-741.03)$

$12.80(4.09-40.03)$

$4.49(1.76-11.44)$

$2.95(1.38-6.30)$

Random effects model

Pooled diagnostic OR $=9.10(5.43-15.25)$

Cochran- $Q=24.44 ; d f=9(P=0.0037)$

sistency $\left(I^{2}\right)=63.2 \%$

$\tau^{2}=0.4161$

Figure 3 Forest plot (random effects model) of pooled positive likelihood ratio, negative likelihood ratio, diagnostic odds ratio for diagnosis of ovarian cancer. Notes: (A) Positive likelihood ratio; (B) negative likelihood ratio; and (C) diagnostic odds ratio.

Abbreviations: $\mathrm{Cl}$, confidence interval; LR, likelihood ratio; OR, odds ratio. 


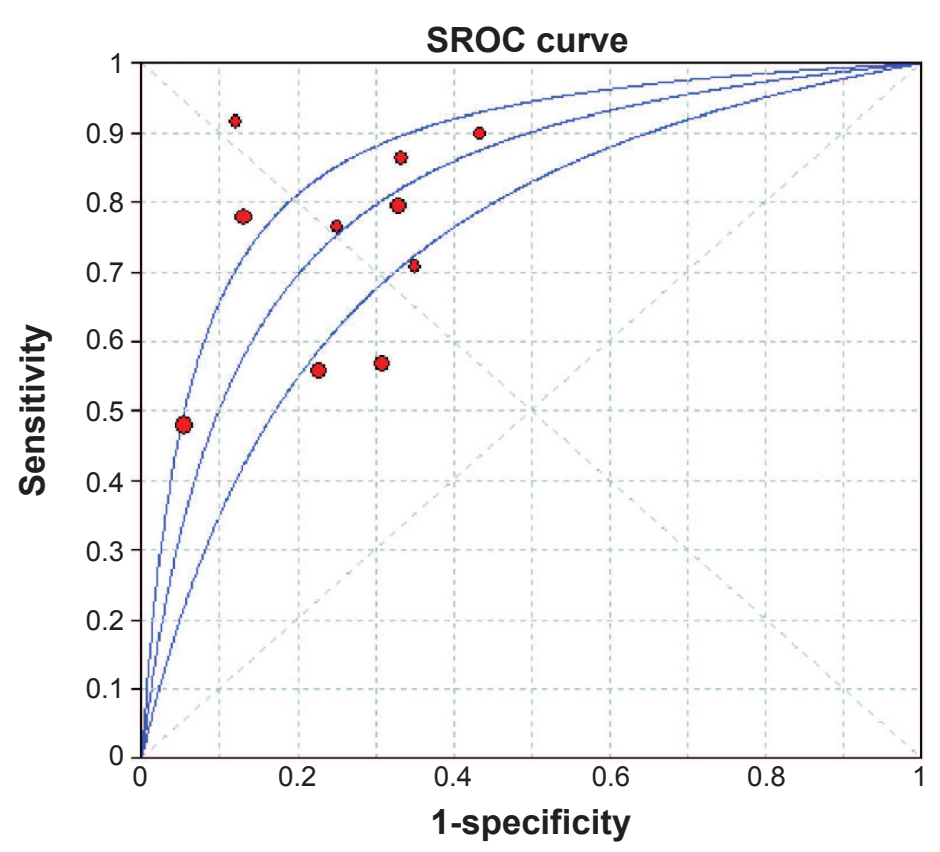

Symmetric SROC

AUC $=0.8175$

SE $(A \cup C)=0.0287$

$\mathrm{Q}^{*}=0.7513$

SE $\left(Q^{*}\right)=0.0257$

Figure 4 Summary receiver operating characteristic (SROC) curve for diagnosis of ovarian cancer. Abbreviations: AUC, area under the curve; SE, standard error.

the selected studies by the $Q$-test and $I^{2}$ statistic of inconsistency analysis; therefore, we used random effect models to calculate SEN, SPE, PLR, and NLR, DOR, and SROC. The threshold effect is a primary cause of heterogeneity in test accuracy studies, but Spearman correlation coefficient was $0.312(P=0.365)$ in the present meta-analysis, suggesting that threshold effect may not be a heterogeneity source of this meta-analysis. In order to explore the potential source of heterogeneity, we performed subgroup analyses according to ethnicity and sample size. Moreover, the subgroup analysis showed that diagnostic performance of VEGF in an Asian population was better than that in the Caucasian population, indicating that the value of VEGF in the diagnosis of OC may be different between races. However, the subgroup analyses results showed that the above-mentioned factors do not significantly affect heterogeneity, suggesting that the influencing factors are complex.

There were still some limitations in our meta-analysis. First, VEGF is a recently discovered biomarker, so few studies were available for our meta-analysis; therefore, our results might change as more studies are performed with VEGF. Second, the controls included in our study proved to be quite heterogeneous. Different studies used different controls, such as healthy individuals and benign disease. The uniform control groups must be established so that the accuracy of circulating VEGF as a diagnostic tool may not be overestimated. Third, the number of studies included in this meta-analysis was limited; therefore, additional studies are needed to assess the diagnostic accuracy of VEGF in the future.

Table 2 Subgroup analysis for diagnostic accuracy of VEGF for ovarian cancer

\begin{tabular}{|c|c|c|c|c|c|c|}
\hline $\begin{array}{l}\text { Subgroup } \\
\text { analysis }\end{array}$ & SEN (95\% Cl) & SPE $(95 \% \mathrm{Cl})$ & PLR (95\% Cl) & NLR $(95 \% \mathrm{Cl})$ & DOR (95\% Cl) & AUC \\
\hline Overall & $0.67(0.63-0.7 \mathrm{I})$ & $0.78(0.75-0.8 \mathrm{I})$ & $3.08(2.27-4.19)$ & $0.39(0.29-0.5 \mathrm{I})$ & $9.10(5.43-15.25)$ & 0.8175 \\
\hline \multicolumn{7}{|l|}{ Ethnicity } \\
\hline Asian & $0.82(0.75-0.88)$ & $0.77(0.7 \mathrm{I}-0.82)$ & $3.12(1.97-4.94)$ & $0.25(0.18-0.36)$ & 14.95 (8.76-25.50) & 0.8705 \\
\hline Caucasian & $0.60(0.54-0.65)$ & $0.79(0.75-0.83)$ & $3.11(1.99-4.85)$ & $0.5 \mathrm{I}(0.4 \mathrm{I}-0.64)$ & $7.00(3.58-13.70)$ & 0.7814 \\
\hline \multicolumn{7}{|l|}{ Sample size } \\
\hline$\geq 100$ & $0.60(0.55-0.66)$ & $0.81(0.77-0.85)$ & $3.41(2.00-5.79)$ & $0.47(0.35-0.63)$ & $8.11(3.75-17.54)$ & 0.7832 \\
\hline$<100$ & $0.8 \mathrm{I}(0.74-0.87)$ & $0.72(0.65-0.78)$ & $2.78(1.93-4.01)$ & $0.28(0.18-0.45)$ & $10.33(5 .||-20.87)$ & 0.8113 \\
\hline
\end{tabular}

Abbreviations: SEN, sensitivity; SPE, specificity; PLR, positive likelihood ratio; NLR, negative likelihood ratio; DOR, diagnostic odds ratio; AUC, area under curve; $\mathrm{Cl}$, confidence interval. 


\section{Conclusion}

In summary, the present meta-analysis suggests that circulating VEGF has moderate diagnostic accuracy for OC. Considering our limitations and the heterogeneity among our selected studies, larger, well-designed prospective and multicenter validation studies are needed to evaluate the diagnostic value of circulating VEGF for OC.

\section{Disclosure}

The authors declare that they have no competing interests.

\section{References}

1. Jemal A, Bray F, Center MM, Ferlay J, Ward E, Forman D. Global cancer statistics. CA Cancer J Clin. 2011;61(2):69-90.

2. Bowtell DD. The genesis and evolution of high-grade serous ovarian cancer. Nat Rev Cancer. 2010;10(11):803-808.

3. Bast RC Jr, Hennessy B, Mills GB. The biology of ovarian cancer: new opportunities for translation. Nat Rev Cancer. 2009;9(6):415-428.

4. Lichtman SM. How I treat ovarian cancer in older women. $J$ Geriatr Oncol. 2014;5(3):223-229.

5. Pradeep CR, Sunila ES, Kuttan G. Expression of vascular endothelial growth factor (VEGF) and VEGF receptors in tumor angiogenesis and malignancies. Integr Cancer Ther. 2005;4(4):315-321.

6. Goel HL, Mercurio AM. VEGF targets the tumour cell. Nat Rev Cancer. 2013;13(12):871-882.

7. Kondo S, Asano M, Matsuo K, Ohmori I, Suzuki H. Vascular endothelial growth factor/vascular permeability factor is detectable in the sera of tumor-bearing mice and cancer patients. Biochim Biophys Acta. 1994;1221(2):211-214.

8. Kushlinskii NE, Gershtein ES, Nikolaev AA, et al. Insulin-like growth factors (IGF), IGF-binding proteins (IGFBP), and vascular endothelial growth factor (VEGF) in blood serum of patients with colorectal cancer. Bull Exp Biol Med. 2014;156(5):684-688.

9. Fu ZZ, Sun XD, Li P, et al. Relationship between serum VEGF level and radiosensitivity of patients with nonsmall cell lung cancer among asians: a meta-analysis. DNA Cell Biol. 2014;33(7):426-437.

10. Wu J, Liu X, Wang Y. Predictive value of preoperative serum CCL2, CCL18, and VEGF for the patients with gastric cancer. BMC Clin Pathol. 2013;13:15.

11. Dobrzycka B, Terlikowski SJ, Kowalczuk O, Kulikowski M, Niklinski J. Serum levels of VEGF and VEGF-C in patients with endometrial cancer. Eur Cytokine Netw. 2011;22(1):45-51.

12. Coskun U, Gunel N, Toruner FB, et al. Serum leptin, prolactin and vascular endothelial growth factor (VEGF) levels in patients with breast cancer. Neoplasma. 2003;50(1):41-46.

13. Vysotskii MM, Digaeva MA, Kushlinskii NE, et al. Serum sFas, leptin, and VEGF in patients with ovarian cancer and benign tumors. Bull Exp Biol Med. 2009;148(5):810-814.
14. Whiting P, Rutjes AW, Reitsma JB, Bossuyt PM, Kleijnen J. The development of QUADAS: a tool for the quality assessment of studies of diagnostic accuracy included in systematic reviews. BMC Med Res Methodol. 2003;3:25.

15. Egger M, Davey Smith G, Schneider M, Minder C. Bias in meta-analysis detected by a simple, graphical test. BMJ. 1997;315(7109):629-634.

16. Robati M, Ghaderi A, Mehraban M, Shafizad A, Nasrolahi H, Mohammadianpanah M. Vascular endothelial growth factor (VEGF) improves the sensitivity of CA125 for differentiation of epithelial ovarian cancers from ovarian cysts. Arch Gynecol Obstet. 2013;288(4): 859-865.

17. Lawicki S, Bedkowska GE, Gacuta-Szumarska E, Szmitkowski M. The plasma concentration of VEGF, HE4 and CA125 as a new biomarkers panel in different stages and sub-types of epithelial ovarian tumors. J Ovarian Res. 2013;6(1):45.

18. Qu FF LH, Zhao JG. The Expression and Significance of VEGF and sFlt1 in Epithelial Ovarian Cancer. Chinese Journal of Clinical Oncology. 2006;33(19).

19. Harlozinska A, Sedlaczek P, Kulpa J, et al. Vascular endothelial growth factor (VEGF) concentration in sera and tumor effusions from patients with ovarian carcinoma. Anticancer Res. 2004;24(2C):1149-1157.

20. Li L, Wang L, Zhang W, et al. Correlation of serum VEGF levels with clinical stage, therapy efficacy, tumor metastasis and patient survival in ovarian cancer. Anticancer Res. 2004;24(3b):1973-1979.

21. Gorelik E, Landsittel DP, Marrangoni AM, et al. Multiplexed immunobead-based cytokine profiling for early detection of ovarian cancer. Cancer Epidemiol Biomarkers Prev. 2005;14(4):981-987.

22. Tanir HM, Ozalp S, Yalcin OT, Colak O, Akcay A, Senses T. Preoperative serum vascular endothelial growth factor (VEGF) in ovarian masses. Eur J Gynaecol Oncol. 2003;24(3-4):271-274.

23. Tang XM YW, Ma LY. Diagnostic value of serum VEGF, Ca125, SA, CEA, AFP in ovarian cancer. Tumor. 2003;23(3).

24. Oehler MK, Caffier H. Diagnostic value of serum VEGF in women with ovarian tumors. Anticancer Res. 1999;19(4A):2519-2522.

25. Obermair A, Tempfer C, Hefler L, et al. Concentration of vascular endothelial growth factor (VEGF) in the serum of patients with suspected ovarian cancer. Br J Cancer. 1998;77(11):1870-1874.

26. Du K, Gong HY, Gong ZM. Influence of serum VEGF levels on therapeutic outcome and diagnosis/prognostic value in patients with cervical cancer. Asian Pac J Cancer Prev. 2014;15(20):8793-8796.

27. Zhen S, Bian LH, Chang LL, Gao X. Comparison of serum human epididymis protein 4 and carbohydrate antigen 125 as markers in ovarian cancer: A meta-analysis. Mol Clin Oncol. 2014;2(4):559-566.

28. Glas AS, Lijmer JG, Prins MH, Bonsel GJ, Bossuyt PM. The diagnostic odds ratio: a single indicator of test performance. $J$ Clin Epidemiol. 2003;56(11):1129-1135.

29. Walter SD. Properties of the summary receiver operating characteristic (SROC) curve for diagnostic test data. Stat Med. 2002; 21(9):1237-1256.
OncoTargets and Therapy

\section{Publish your work in this journal}

OncoTargets and Therapy is an international, peer-reviewed, open access journal focusing on the pathological basis of all cancers, potential targets for therapy and treatment protocols employed to improve the management of cancer patients. The journal also focuses on the impact of management programs and new therapeutic agents and protocols on
Dovepress

patient perspectives such as quality of life, adherence and satisfaction. The manuscript management system is completely online and includes a very quick and fair peer-review system, which is all easy to use. Visit http://www.dovepress.com/testimonials.php to read real quotes from published authors. 